\title{
Tangence
}

\section{La formation d'une chroniqueuse : Mémoires d'une jeune fille rangée}

\section{Marylea MacDonald}

Numéro 45, octobre 1994

Authenticité et littérature personnelle

URI : https://id.erudit.org/iderudit/025829ar

DOI : https://doi.org/10.7202/025829ar

Aller au sommaire du numéro

Éditeur(s)

Tangence

ISSN

0226-9554 (imprimé)

1710-0305 (numérique)

Découvrir la revue

Citer cet article

MacDonald, M. (1994). La formation d'une chroniqueuse : Mémoires d'une jeune

fille rangée. Tangence, (45), 107-115. https://doi.org/10.7202/025829ar d'utilisation que vous pouvez consulter en ligne.

https://apropos.erudit.org/fr/usagers/politique-dutilisation/ 


\section{La formation d'une chroniqueuse : Mémoires d'une jeune fille rangée Marylea MacDonald}

Dès le premier tome de ses Mémoires, Simone de Beauvoir construit non seulement l'histoire de sa propre vie, qu'elle veut à la fois unique et représentative, mais aussi celle d'une époque. Dans sa tentative pour cerner son temps, Julia Kristeva tient Beauvoir pour "a chronicler who knew how to construct an entire cultural phenomenon " ${ }^{1}$. En son origine médiévale, la chronique prétendait relater les événements historiques selon l'ordre de leur déroulement, le plus souvent sans commentaire. Néanmoins, nous savons maintenant que l'ordre adopté et le choix des événements constituent déjà un commentaire. Sous la plume d'un romancier, d'un Stendhal ou d'un Duhamel, la chronique met en scène des personnages réels ou fictifs, tout en évoquant des faits sociaux et historiques authentiques. L'ordre chronologique du récit et sa stricte véracité sont affectés par une intervention explicite du chroniqueur.

Maints facteurs se réunissaient pour offrir à Beauvoir la distance et l'intimité voulues pour établir la chronique de son époque. En tant qu'intellectuelle, elle côtoyait des personnalités engagées politiquement, sans partager leur désir d'agir, sauf par l'écriture. En tant que femme, elle n'était pas assujettie à certaines obligations, comme la mobilisation en temps de guerre, mais elle n'a épargné aucun effort pour connaître la vie de soldat et pour comprendre la guerre. Son Journal de guerre témoigne de ses dons pour l'observation et de son peu d'intérêt pour l'analyse introspective. À partir de La force de l'âge, ses Mémoires construisent l'avant-guerre, la guerre, l'après-guerre et les guerres coloniales. Les mandarins seront l'occasion pour Beauvoir d'exceller dans la construction fictive du phénomène de l'après-guerre.

La présente étude tente de réfléchir à quelques stratégies narratives reconnaissables dans le récit autobiographique, à la fois

1 Julia Kristeva, "My Memory's Hyperbole", The Female Autograph, Domna C. Stanton (éd.), Chicago, The University of Chicago Press, 1987, p. 219. 
108

authentique et associé à la fiction, des Mémoires d'une jeune fille rangée ${ }^{2}$, le tome qui s'attache à la formation de Beauvoir, chroniqueuse. En premier lieu, j'introduirai une comparaison entre la structure du temps dans le récit et le temps réel. Beauvoir était consciente des difficultés langagières inhérentes à l'autobiographie, et elle a essayé d'y porter remède. Dans le deuxième volet de cette étude, je considérerai certaines des stratégies adoptées: structure cyclique, mirages optiques et constitution du personnage.

Une comparaison du temps dans le récit et du temps réel permet de déceler des décalages. Le premier se manifeste au niveau de l'organisation du livre en quatre parties dont chacune couvre une durée de plus en plus rétrécie: $1^{\text {re }}$ partie: 11 ans; $2^{\mathrm{e}}$ partie : 6 ans; $3^{\mathrm{e}}$ partie: 3 ans; $4^{\mathrm{e}}$ partie: 1 an. La longueur de chaque partie est variable: $124,101,157$ et 110 pages, respectivement. L'espace du texte n'entretient pas un rapport de proportionnalité avec la durée du temps réel. Pourquoi Beauvoir a-t-elle divisé son livre en quatre parties et pourquoi a-t-elle choisi ces articulations-là? Dans La force de l'âge, avant d'entamer une justification de ce qu'elle appelle "une ère nouvelle", Beauvoir nous dit qu' [i]l est arbitraire de découper sa vie en tranches" 3 . Ici elle ne nous a pas laissé de titres pour nous guider, chaque section portant un simple chiffre romain. Puisque les intentions de cette autobiographie ne nous sont pas discernables, nous pouvons seulement observer que la structure temporelle adoptée correspond à la mémoire plutôt qu'au temps réel. Son enfance lointaine est racontée en moins d'espace, car elle en a moins de souvenirs. "De mes premières années, je ne retrouve guère qu'une impression confuse: quelque chose de rouge, et de noir, et de chaud." (p. 9) Au fur et à mesure qu'elle avance dans son récit, les souvenirs se font de plus en plus nombreux. Les Mémoires dépendent plus de la mémoire que des événements réels. Les interventions du "je" narratrice dans son récit pour attester la véracité de ses souvenirs témoignent de la conscience chez Beauvoir de la fonction de la mémoire, comme dans

2 Simone de Beauvoir, Mémoires d'une jeune fille rangée, Paris, Gallimard, coll. "Folio, 1958. Les références à cette œuvre seront dorénavant indiquées dans le texte entre parenthèses.

3 Simone de Beauvoir, La force de l'âge, Paris Gallimard, coll. "Folio", 1960, p. 410 . 
la première remarque citée ci-dessus: "je ne retrouve guère qu'une impression confuse" (p. 9).

Quelle est la structure temporelle du passé de la mémoire? Que devient-elle quand on l'introduit dans un récit? Quels rapports existe-t-il entre le réel, c'est-à-dire le vécu au moment où on le vit, le souvenir du réel, et le récit du souvenir du réel?

La structure temporelle du premier tome des Mémoires de Beauvoir est chronologique. Les grandes périodes des vingt premières années de sa vie - enfance, adolescence, et années de jeune fille - se succèdent dans le récit dans l'ordre dans lequel elle les a vécues ${ }^{4}$. Beauvoir maintient cet ordre chronologique dans les deux tomes suivants, La force de l'âge et La force des choses. Elle renonce à l'ordre chronologique pour l'ordre thématique dans Tout compte fait, pour les raisons suivantes: "aucun événement public ni privé n'a profondément modifié ma situation: je n'ai pas changé " 5 . L'ordre chronologique fait du récit une mimésis de la vie, mais la matière de l'imitation impose des déformations. "En la contenant dans des phrases, nous dit-elle, mon récit fait de mon histoire une réalité finie, qu'elle n'est pas. Mais aussi il l'éparpille, la dissociant en un chapelet d'instants figés, alors qu'en chacun passé, présent et avenir étaient indissolublement liés. " 6 Elle regrette d'avoir "échoué à donner aux heures révolues leur triple dimension " ${ }^{7}$. Alors pourquoi n'a-t-elle pas cherché d'autres ordres de présentation de son existence? Dans La force des choses, nous apprenons que la raison qui a commandé le choix de Beauvoir est le rôle du temps dans sa perception de sa vie: "ce qui compte avant tout dans ma vie, c'est que le temps coule; je vieillis, le monde change, mon rapport avec lui varie; montrer les transformations, les mûrissements, les irréversibles dégradations des autres et de moi-même, rien ne m'importe davantage. Cela m'oblige à suivre docilement le fil des années" 8 . Beauvoir reprend cette explication dans le tome suivant: "Vivre

4 "C'est pourquoi les Mémoires d'une jeune fille rangée ont une unité romanesque qui manque dans les volumes suivants, observe-t-elle. Comme dans les romans d'apprentissage, du début à la fin le temps coule avec rigueur." Simone de Beauvoir, Tout compte fait, Paris, Gallimard, coll. "Folio", 1972, p. 27.

5 Ibid., p. 10.

6 Ibid., p. 9.

7 Ibid., p.10.

8 Simone de Beauvoir, La force des choses, Paris, Gallimard, 1963, p. 296-297. 
était pour moi une entreprise clairement orientée et pour en rendre compte il me fallait en suivre le cheminement. "?

Rédiger son autobiographie dans l'ordre chronologique revient à une décision philosophique pour Beauvoir, mais le récit apporte des obstacles à la réussite de son projet. La réalisation de son désir de raconter l'histoire de sa vie à sa façon entraîne des conséquences subversives. L'histoire racontée ne correspond pas à la vie vécue. Plus elle poursuit son projet, plus le projet lui échappe. Il me semble que Beauvoir a adopté des stratégies narratives qui devaient effacer l'écart entre le récit et la vie, qui devaient rapprocher la vie du récit, mais d'un certain type de récit, celui de la chronique. Ainsi interviennent la structure cyclique, les mirages optiques et la création du personnage.

Dans les chansons de geste, prototype des chroniques fictives, le narrateur reprend à maintes reprises le même événement. Ainsi lisons-nous plusieurs fois l'histoire de telle matinée ou de telle bataille. La tradition veut que cette caractéristique ait son origine dans le caractère oral de la chanson de geste. Cependant, une variante de ce phénomène dans les Mémoires de Beauvoir suggère une fonction plus profonde de la structure cyclique. Quand la geste se transforme en res gestce, la narration imite les conventions de la chanson.

Prenons comme exemple le récit du début de sa carrière d'écrivain. La narratrice des Mémoires d'une jeune fille rangée ne fournit pas un seul, mais plusieurs débuts. De son premier désir "d'inverser [la] magie" "[d]es signes imprimés en récit" (p. 72), réalisé dans Les malbeurs de Marguerite et La famille Fenouillard, les œuvres de son enfance, au plaisir qu'elle prenait à faire ses "compositions françaises" (p. 96), ses rapports à l'écriture se composent peu à peu. À mi-chemin du récit, la narratrice s'interroge: "Pourquoi ai-je choisi d'écrire?" (p. 196) En guise de réponse, elle retrace l'histoire de ses "gribouillages" enfantins et de ses exercices scolaires, pour aboutir à la réponse qu'elle a donnée à quinze ans à la question posée dans l'album d'une amie, "Que voulezvous faire plus tard?": "Être un auteur célèbre." (p. 196) Ensuite la narratrice se lance dans une analyse, faite au moment de la rédaction, de cette déclaration. Les raisons qu'elle relève sont "l'admiration que [lui] inspiraient les écrivains", "le goût de la

9 Tout compte fait, p. 10. 
communication" et son rêve "d'être [s]a propre cause et [s]a propre fin" (p. 196-197). Dans la deuxième moitié du volume, la narratrice raconte les romans que Simone, étudiante, a entrepris, tout en indiquant les rapports qu'y entretiennent l'auteure et ses personnages. "Sous le nom d'Éliane, je me promenais dans un parc avec des cousins, des cousines" (p. 265). "Je composai ma première ouuvre. C'était l'histoire d'une évasion manquée. L'héroïne avait mon âge, dix-huit ans" (p. 288). "Je commençai un vaste roman; l'héroïne traversait toutes mes expériences" (p. 349). Il est à remarquer que chaque fois Beauvoir commence un autre roman et que les premières œuvres se multiplent. À la différence de la chanson de geste, il ne s'agit pas du même événement ou du même moment, mais plutôt d'un phénomène achronique qu'elle insère dans un récit chronologique. Que signifient ces nombreux débuts, ce retour cyclique à une tentative de préciser l'origine de son écriture? J'y vois le véritable caractère d'une origine: multiple. À force de se répéter, un phénomène finit par confirmer son origine et la dépasser. Aussi, l'origine se fixe dans le récit, pour la narratrice et pour le lecteur. La mémoire d'un lecteur n'est peut-être pas différente de celle de la foule qui entend déclamer une chanson de geste. Le retour au site de l'origine a un autre effet aussi; il rehausse la valeur du phénomène raconté. Le rythme du retour, avec ses appels aux rites religieux et naturels, consacre l'origine de l'écriture, l'investissant d'une valeur sacrée.

Béatrice Didier essaie de cerner dans le temps cyclique ce qui serait propre à l'expérience féminine du temps.

Il est possible aussi que la femme ressente le temps autrement que ne le fait l'homme, puisque son rythme biologique est spécifique. Temps cyclique, toujours recommencé, mais, avec ses ruptures, sa monotonie et ses discontinuités.[...] Peut-être pour la femme, plus que pour l'homme, le temps est-il perceptible en dehors de l'événement, parce qu'elle porte en elle ses propres événements. ${ }^{10}$

Tout en avouant sa méfiance devant la construction d'archétypes d'écriture et d'expérience féminines, Didier interprète "la prolixité féminine" comme "le signe d'une créativité débordante et des

10 Béatrice Didier, L'écriture-femme, Paris, Presses universitaires de France, 1981 , p. 32-33. 
112

forces vives de la femme accédant à l'écriture" et "le moyen de rendre cette conscience du temps" 11 .

Beauvoir adopte une structure cyclique également dans son traitement des personnages de son récit autobiographique. Nous rencontrons ses parents et sa gouvernante, Louise, déjà dans les premières pages. La narratrice consacre un paragraphe à chacun des trois pour dire quel rôle ils ont joué dans son enfance. Ses parents et Louise reviennent au cours des pages suivantes, soit pour l'explication de leur rôle, soit dans un épisode où ils figurent. La narratrice aurait pu achever là son portrait de ses parents, mais à la page 45, elle écrit: "Il est temps de dire, dans la mesure où je le sais, qui ils étaient. " N'entre en jeu que ce qu'elle sait, et pas ici son point de vue. Évidemment, les deux questions sont reliées, mais Beauvoir semble prendre son portrait de ses parents pour le seul vrai portrait possible, refusant d'avance le droit de parole à ses parents, ainsi qu'à autrui. L'occasion de ce portrait est fournie par une réflexion sur son identité de petite fille: "Pendant plusieurs années, je me fis le docile reflet de mes parents " (p. 44-45). D'autres personnages sont présentés de la même manière, notamment Jacques et Zaza, les amis de son enfance. De leur première apparition dans le récit, c'est-à-dire dans la vie de Beauvoir, jusqu'à leur disparition définitive, et dans les deux cas par la mort, ces deux personnes font des retours cycliques dans lesquels Beauvoir essaie de mettre au clair ses rapports avec eux. Encore une fois aucune nécessité d'intrigue ne commande cette pluralité de retours. Le récit aurait pu se construire d'une autre manière et les lecteurs n'en auraient pas souffert. La structure cyclique, voire répétitive, doit ses origines au style de narration de Beauvoir, à un style qui rappelle les cycles de genres disparus il y a longtemps, ou transformés en d'autres genres.

Si la structure cyclique nous rappelle les stratégies d'une littérature orale, les mirages optiques font appel à un art visuel. Le modèle s'inspire de jouets préférés que Beauvoir décrit dans les Mémoires d'une jeune fille rangée. Les seuls jouets qui la "captivèrent " étaient le stéréoscope, le kinétoscope, et "des espèces d'albums qu'on animait d'un simple coup de pouce" (p. 34). "Jeux d'ombres, projections lumineuses: ce qui m'intéressait dans tous les mirages optiques, c'est qu'ils se composaient et se recom-

11 Ibid., p. 33. 
posaient sous mes yeux."(p. 34) Sa fascination devant le processus indéfini de recomposition annonce son grand intérêt pour la mobilité complexe du monde. Lorsque Beauvoir se met à apprendre, elle compare le monde à "un album d'images aux couleurs brillantes que je feuilletais, dit-elle, avec ravissement" (p. 33). Raconter l'histoire d'une seule vie exige qu'on tienne compte de plusieurs vies. Dans le texte, les mirages optiques se retrouvent dans une pluralité de points de vue et de digressions. Beauvoir change de point de vue en donnant la parole à quelqu'un d'autre, en intégrant d'autres documents dans ses Mémoires: son journal intime de jeune fille, des citations et des lettres. Et elle abandonne le récit de sa vie pendant des pages pour raconter la vie d'autres gens. Elle justifie ces digressions à partir de son récit matriciel, mais elle semble partager avec George Sand le plaisir en soi de la digression ${ }^{12}$. Raconter à côté et autour de son personnage est une autre manière de composer et de recomposer son récit.

Sa fascination la plus profonde demeure cependant attachée à sa personne, à tel point que son désir de se regarder vivre et de dire cette vie finit par transformer sa personne en personnage. Alors que Sartre était "The Boy Who Wanted to Be a Book" 13, selon le dire de Paul Eakin, Beauvoir se contentait de devenir le personnage principal dans la chronique de sa vie et de son époque. On pourrait donner le nom de personnagification au processus de transformation d'une personne en personnage, un mot qui regroupe la fin et les moyens. Le terme s'impose aussi à cause des rapports d'identité que Beauvoir établit avec son personnage. Elle s'identifie avec son personnage comme un lecteur s'identifie avec un personnage fictif. Une conséquence, positive pour elle, en est la catharsis de l'écriture autobiographique. La résistance de Beauvoir à une contestation de son personnage témoigne à la fois de sa réticence devant une remise en question de son histoire et de sa certitude que son autoportrait est fidèle. Dans le paratexte elle insiste sur la vérité de ce qu'elle dit, tout en reconnaissant l'impossibilité de dire tout.

12 Au sujet de sa première digression dans le premier chapitre de son autobiographie, George Sand nous dit: "je me suis permis une fois pour toutes les interminables digressions". George Sand, Histoire de ma vie, Euvres autobiographiques, vol. I, Paris, Gallimard, "Bibliothèque de la Pléiade”, 1970, p. 18.

13 Paul John Eakin, Fictions in Autobiograpby: Studies in the Art of Self-Invention, Princeton, Princeton University Press, 1988, p. 126. 
114

La personnagification prend son point de départ dans les rapports qu'entretenait déjà la petite fille avec l'écriture, rapports dont elle attribue l'origine à ses parents. C'est dans une carte postale envoyée par un ami de son père, que le prénom de l'auteure figure dans le récit pour la première fois: "Simone aime-t-elle toujours la salade cuite?» (p. 14) Sa réaction est à noter: “L'écriture avait à mes yeux plus de prestige encore que la parole: j'exultai." (p. 14) Toutefois le plaisir de l'intérêt manifesté par autrui ne va pas sans déception. Quand elle revoit l'auteur de la carte postale, elle essaie de solliciter une réaction: "il n'y eut pas d'écho" (p. 14). Après s'être plu à se contempler dans l'image qu'autrui avait construite d'elle-même, elle se retrouve dans le vide de la solitude. "Je découvris avec dépit combien la gloire est éphémère." (p. 14) L'image textuelle suivante de la petite Simone est le récit d'une de ses "crises furieuses" (p. 17) écrit par "une tante obèse et moustachue, qui maniait la plume "(p. 18). Ce portrait peu flatteur de l'auteure prépare l'échec du récit. Par contre son titre, La poupée modèle, annonce le titre de ses Mémoires. La leçon de l'ironie est cachée. "[Ā] travers le récit que me lisait Louise, je me sentis un personnage; peu à peu cependant, la gêne me gagna." (p. 18) La vie d'un personnage, comme Simone l'a déjà découvert, comporte des hauts et des bas, gloire et déception. De cette rencontre avec son image textuelle fournie par autrui, elle tire la leçon suivante: "la littérature ne soutient avec la vérité que d'incertains rapports" (p. 18). Les écarts entre l'histoire fictive et la vérité sont dans les larmes attribuées à sa gouvernante, Louise, "qui ne pleurait jamais" et dans la métaphore des brebis employée pour évoquer les enfants: "comment peut-on comparer une petite fille à des moutons?» (p. 18) Beauvoir adopte le point de vue de la petite Simone pour raconter ces épisodes.

Le processus d'élaboration de son personnage répond à une tentative pour se fixer sur le papier. Après ces premières rencontres avec les images des autres, Beauvoir commence à créer ses propres images. Aussi, très jeune, elle se met à tenir un journal intime. Vivre et se raconter. "Ma vie serait une belle histoire qui deviendrait vraie au fur et à mesure que je me la raconterais" (p. 234).

Une personne en train de se faire personnage entretient des rapports privilégiés avec d'autres personnages, surtout fictifs, du moins déjà fixés par l'écriture. Ainsi peut se comprendre 
l'influence énorme de lectures de Beauvoir sur son imaginaire. Que cela soit Little Women ou Le moulin sur la Floss, la jeune fille a dévoré les histoires de Joe et de Meg, de Maggie Tulliver, toujours à la recherche de sa propre histoire. Elle s'est reconnue dans ces personnages. À partir de ces récits qui lui fournissaient d'autres modèles de vie, elle cherchait ce qu'elle allait devenir. Le futur se déchiffrait dans les livres et s'inventait dans sa vie imaginaire: "Je me crus autorisée moi aussi à considérer mon goût pour les livres, mes succès scolaires, comme le gage d'une valeur qui confirmerait mon avenir. Je devins à mes propres yeux un personnage de roman." (p. 124) À la fin de Tout compte fait, Beauvoir revient à son identification à ces personnages: “ j'ai souhaité revêtir moi-même, aux yeux d'un public, cette dimension imaginaire qui rendait pour moi si fascinantes ces héroïnes de roman et l'auteur qui se projetait en elle. [...] plus tard, j'ai tenté de me raconter, dotant mon expérience d'une nécessité "14.

Chez Beauvoir, raconter sa vie revient à une manie pourvue d'un caractère épistémologique. "[O]n ne peut jamais se connaître, affirme-t-elle, mais seulement se raconter." 15 Ou encore, "Icle qui m'aide à réfléchir sur [ma vie], c'est que je l'ai racontée. "16 Malgré l'impossibilité de coïncidence entre le récit autobiographique et le réel ${ }^{17}$, malgré l'écart qui sépare ses souvenirs et leur résurrection "en noir et blanc" "18, son désir de faire une chronique de sa vie et de son époque est irrépressible. "Construire une image de moi-même: cette vaine et d'ailleurs impossible entreprise ne m'intéresse pas. Ce que je souhaiterais c'est me faire une idée de ma situation dans le monde." ${ }^{19}$ Une fois "lancée dans [l']imprudente aventure " 20 avec les Mémoires d'une jeune fille rangée, Beauvoir fera des retours multiples à "cet étrange objet qu'est une vie ${ }^{21}$ jusqu'à en construire un phénomène culturel.

14 Tout compte fait, p. 634.

15 La force de l'âge, p. 422.

16 Tout compte fait, p. 13.

17 «D'accord: le récit se déroule sur un autre terrain que l'expérience vécue; mais il s'y réfère et peut permettre d'en dégager certains traits. "Ibid., p. 13.

18 La force de l'âge, p. 9.

19 Tout compte fait, p. 58.

20 La force de l'âge, p. 9.

21 Tout compte fait, p. 9. 